\title{
ATENÇÃO À MULHER MASTECTOMIZADA: DISCUTINDO OS ASPECTOS ÔNTICOS E A DIMENSÄO ONTOLÓGICA DA ATUACÃO DA ENFERMEIRA NO HOSPITAL DO CÂNCER IIIT
}

\author{
Teresa Caldas Camargo ${ }^{2}$ \\ Ivis Emília de Oliveira Souza ${ }^{3}$
}

Camargo TC, Souza IEO. Atenção à mulher mastectomizada: discutindo os aspectos ônticos e a dimensão ontológica da atuação da enfermeira no hospital do câncer III. Rev Latino-am Enfermagem 2003 setembro-outubro; 11(5):614-21.

Esta reflexão aborda os aspectos relativos às dimensões ôntica e ontológica, que constituem e, portanto, permeiam o cenário da atuação da enfermeira junto à cliente com câncer de mama, submetida à mastectomia. A análise reflexiva foi desenvolvida considerando tanto a experiência profissional como enfermeira assistencial do Hospital do Câncer III (HC III) do Instituto Nacional de Câncer (INCA), como pelo apoio de um referencial teórico filosófico obtido ao cursar o mestrado e o doutorado em Enfermagem. Numa atitude compreensiva e de interpretação fenomenológica, pretende-se refletir sobre as ações assistenciais da enfermeira na rotina do espaço institucional e apontar possibilidades da ação assistencial a partir da dimensão existencial das pessoas envolvidas. Nesse sentido, ênfase é dada à atuação da enfermeira junto à cliente, que não se traduz apenas na aplicação de técnicas científicas, orientações e informações, mas também percebe, compreende e cuida da mulher cidadã em sua singularidade.

DESCRITORES: neoplasias mamárias; cuidados de enfermagem; enfermagem oncológica

\section{CARE TO MASTECTOMIZED WOMAN: DISCUSSING ONTIC ASPECTS AND THE ONTOLOGICAL DIMENSION IN NURSES PERFORMANCE AT A CANCER HOSPITAL}

This reflection approaches aspects related to the ontic and ontological dimensions that constitute and, therefore, permeate nursing care delivered to the client with breast cancer submitted to mastectomy. This reflexive analysis was developed considering the author's professional experience as a clinical nurse at the Cancer Hospital III and at the National Cancer Institute (INCA) as well as using the theoretical frameworks studied during her master and doctoral courses. Through a comprehensive attitude and using phenomenological interpretation, this study aimed at reflecting about nurse's clinical actions in the routine of an institution, pointing out possibilities for a care based on the existential dimension of the involved persons. Therefore, the author emphasizes nurse's performance with the client that is not only translated in the application of scientific techniques, orientations and information but also considering the perception, understanding and care provided by a human being in his/her singularity.

DESCRIPTORS: breast cancer; nursing care; oncology nursing

ATENCIÓN A LA MUJER MASTECTOMIZADA: DISCUTIENDO LOS ASPECTOS ONTICOS Y LA DIMENSION ONTOLÓGICA DE LA ACTUACIÓN DE LA ENFERMERA EN EL HOSPITAL DEL CÁNCER III

Esta reflexión discute los aspectos relacionados con las dimensiones óntica y ontológica, que constituyen y por lo tanto forman el escenario de la actuación de la enfermera con la mujer sometida a la mastectomía. El análisis reflexivo fue desarrollado teniendo en cuenta tanto la experiencia profesional como enfermera asistencial del Hospital del Câncer III (HC III), del Instituto Nacional de Cáncer (INCA), como por el apoyo de un referencial teórico filosófico obtenido durante la maestría y el doctorado en enfermería. Con una actitud comprensiva y de interpretación fenomenológica, se intenta reflejar las acciones asistenciales de la enfermera en el espacio institucional y apuntar las posibilidades de la acción asistencial partiendo de la dimensión existencial de las personas comprometidas. Desde este punto de vista, el énfasis está en la actuación de la enfermera con la mujer mastectomizada, la que no se traduce tan solo en la aplicación de técnicas científicas, orientaciones e informaciones, sino también, percibe, comprende y cuida de la mujer ciudadana en su singularidad.

DESCRIPTORES: neoplasmas de la mama; atención de enfermería; enfermería oncológica

\footnotetext{
${ }_{1}^{1}$ Recorte do trabalho apresentado na mesa técnica "Atenção à mulher mastectomizada" a convite da Sub-Comissão de Temas do 53으르, que condensa as considerações finais da Dissertação de Mestrado e Tese de Doutorado; ${ }^{2}$ Doutor em Enfermagem, Enfermeira da Educação Continuada e Pesquisa de Enfermagem do Hospital do Câncer III (HC III) do Instituto Nacional do Câncer (INCA), Membro da Comissão Científica da Sociedade Brasileira de Enfermagem Oncológica (SBEO), e-mail: tcamargo@inca.gov.br; ${ }^{3}$ Doutor em Enfermagem, Docente da Escola de Enfermagem Anna Nery da Universidade Federal do Rio de Janeiro, Orientadora da Dissertação de Mestrado e da Tese de Doutorado
} 
INTRODUZINDO A TEMÁTICA CÂNCER DE MAMA

$\boldsymbol{O}$ câncer de mama ocupa o segundo lugar no tipo de câncer de maior incidência, estimado para 2001, na população brasileira. A estimativa de casos novos e de óbitos por câncer de mama no Estado do Rio de Janeiro, para 2001, é de 6120 e 1500 casos, respectivamente ${ }^{(1)}$.

O câncer de mama permanece sendo o tipo de tumor mais comum entre as mulheres e tem um típico e, às vezes, complexo impacto psicológico. A utilização, cada vez maior, da cirurgia conservadora (onde não há extirpação completa da mama) tem reduzido o efeito negativo na autoimagem e imagem corporal das mulheres afetadas pela doença ${ }^{(2)}$.

Muitas mulheres hoje, diagnosticadas com câncer de mama, podem ficar curadas ou viver longos períodos com a doença. Entretanto, ao contrário de outros tratamentos para doenças crônicas, aqueles tratamentos contra o câncer são mais tóxicos e intensos, resultando num aumento tanto da demanda das reservas físicas como, também, de seus recursos sociais e psicológicos para sobreviver e enfrentar a doença. Assim, é que o desenvolvimento específico dos cuidados prestados às portadoras de câncer de mama chamou a atenção para o papel chave dos fatores psicossociais na prevenção, no tratamento e nos resultados finais obtidos ${ }^{(3)}$.

O câncer de mama é uma experiência amedrontadora para a mulher e, para maioria delas, o diagnóstico da doença evoca sentimentos de pesar, raiva e intenso medo. Além disso, a doença em sua trajetória, pode levar a mulher a passar por situações que ameaçam sua integridade psicossocial, que provocam incertezas quanto ao sucesso do tratamento e que a levam a se defrontar com a possibilidade de recorrência da doença e a morte ${ }^{(4)}$. Porém, a maioria das mulheres enfrenta a crise e a contorna sem desenvolver desordens psiquiátricas e sexuais severas $^{(2)}$.

A negação e a depressão são as defesas psicológicas geralmente mais utilizadas no caso de acometimento por uma doença como, por exemplo, o câncer de mama. Torna-se, pois, importante a informação adequada sobre a doença e suas conseqüências, pois dá à mulher a possibilidade de enfrentamento e de se adaptar à sua nova condição( ${ }^{(5)}$.

O período cirúrgico é o mais estressante. Apesar da tendência atual para a realização da cirurgia conservadora, muitos casos ainda exigem a mastectomia, e é nessa época que a mulher é confrontada definitivamente com a perda da mama e o medo da cirurgia, da mutilação e da morte ${ }^{(6)}$.

O período pós-operatório é marcado pela ambivalência. Ocorre o alívio de ter sobrevivido à cirurgia e a esperança de estar curada. Mas também há o medo do retorno da doença, o medo de enfrentar a dor e os curativos, o medo de enfrentar a possibilidade permanente de um corpo mutilado e, ainda, preocupações com a feminilidade e com as reações do companheiro frente à mastectomia, podendo esse período levar de um a dois meses após a alta hospitalar. Segue-se, então, o período pós-operatório de dois a seis meses, durante o qual ocorre a depressão, a ansiedade e a diminuição da auto-estima, coexistentes com sentimentos de fortalecimento pessoal e esperança ${ }^{(6)}$.

Assim, o impacto psicossocial do câncer de mama pode ser delineado em três áreas: desconforto psicológico, que causa ansiedade, depressão e raiva; mudanças no estilo de vida, conseqüente ao desconforto físico, disfunção sexual, e alteração do nível de atividade; medo e preocupações com a possibilidade ou a ocorrência da mastectomia, o reaparecimento da doença e a morte ${ }^{(3)}$.

A mastectomia traz para a mulher a realidade da mutilação e com ela um turbilhão de sentimentos. A retirada da mama causa perturbações variadas no cotidiano de muitas mulheres que com ela passam a apresentar nervosismo, agressividade e insegurança. Esses sentimentos parecem permeados de significados como o desespero, medo, aceitação, segurança, impacto, readaptação, intercorrências e ainda a necessidade de autocuidado $^{(7)}$.

O desejo de participação no autocuidado após a cirurgia, em estudo dedicado a analisar as oportunidades e as vontades de mulheres no pós-operatório de câncer de mama, mostrou que, apesar de sua determinação de tomarem parte no autocuidado, infelizmente há pouca informação e suporte profissional para que isso ocorra, o que leva a crer que o sistema de saúde apóia, insuficientemente, a recuperação da paciente. O que as mulheres esperavam após a hospitalização era receber encorajamento, reabilitação mental, ajuda para desenvolver uma atitude positiva em relação à doença e seu tratamento, e apoio dos profissionais de saúde, parentes e amigos ${ }^{(5)}$.

No contato diário com clientes portadoras de câncer de mama, foi observado que as mesmas desejam compartilhar com a enfermeira suas dúvidas, suas 
tristezas, sua desesperança e também suas angústias. $\mathrm{E}$, nesse compartilhar, esperam receber o suporte necessário para enfrentar a doença e o seu tratamento ${ }^{(8)}$.

A assistência de enfermagem em oncologia evoluiu muito desde seu aparecimento como especialidade, e a literatura existente aponta e preconiza importante papel da enfermeira no apoio ao cliente oncológico nas várias fases de sua doença. Pensar hoje em oncologia é pensar em sobrevida com qualidade e não se fixar na cura da doença.

Inicialmente, as enfermeiras oncológicas desempenhavam papéis importantes no cuidado de cabeceira, através de medidas de conforto para os pacientes cirúrgicos e/ou em tratamento paliativo, no caso dos pacientes terminais. Porém, a atuação da enfermeira em oncologia cresceu com o advento dos ensaios clínicos conduzidos com novos agentes terapêuticos quimioterápicos. Esses ensaios clínicos trouxeram a necessidade de um trabalho conjunto da equipe multidisciplinar, voltada para o cuidado do paciente de câncer e para a pesquisa ${ }^{(9)}$.

A assistência de enfermagem em oncologia, então, abrange os vários estágios do continuum saúdedoença, já que, o assistir ao outro que tem câncer, possibilita a intervenção de enfermagem em diversos níveis: na prevenção primária e na prevenção secundária, no tratamento do câncer, na reabilitação e na doença avançada.

Portanto, a prática da enfermeira em oncologia evoluiu para a assistência ao cliente e sua família através da educação, provendo suporte psicossocial, administrando a terapia recomendada, selecionando e administrando intervenções que diminuam os efeitos colaterais da terapia proposta, participando da reabilitação e provendo conforto e cuidado ${ }^{(10)}$.

Esta reflexão aborda os aspectos relativos às dimensões ôntica e ontológica, que constituem e, portanto, permeiam o cenário da atuação da enfermeira junto à cliente com câncer de mama submetida à mastectomia. A análise reflexiva foi desenvolvida considerando tanto a experiência profissional como enfermeira assistencial do Hospital do Câncer III (HC III) do Instituto Nacional de Câncer (INCA), como pelo apoio a um referencial teórico filosófico obtido ao cursar o mestrado e o doutorado em Enfermagem. Numa atitude compreensiva e de interpretação fenomenológica, pretende-se refletir sobre as ações assistenciais da enfermeira na rotina do espaço institucional e apontar possibilidades da ação assistencial a partir da dimensão existencial das pessoas envolvidas.

\section{O ESPAÇO INSTITUCIONAL - A DIMENSÃO ÔNTICA DO ASSISTIR}

O cenário institucional onde atuo, há mais de quinze anos, é o Hospital do Câncer III (HCIII) do Instituto Nacional de Câncer (INCA) que atende, diariamente, uma clientela predominantemente feminina, diagnosticando e tratando pessoas portadoras de câncer de mama e encaminhando, para os locais de atendimento de patologias da mama do município (pólos de mama) as portadoras de doença benigna da mama.

A clientela do hospital vem crescendo dia-a-dia e, com ela, o quantitativo de cirurgias de mama de grande porte (mastectomias e segmentectomias com linfadenectomia axilar), bem como o número de encaminhamentos para o tratamento oncológico, quer seja ele hormonal, quimioterápico e/ou radioterápico.

A porta de entrada para o HC III é a consulta na Triagem, que ocorre diariamente de segunda a sexta-feira. Se nessa consulta for diagnosticada doença benigna da mama, a cliente é encaminhada para os pólos de mama, vinculados à Secretaria Municipal de Saúde; se há suspeita de malignidade ela é matriculada na Instituição, encaminhada para exames complementares que são realizados no mesmo dia da triagem e, na confirmação de malignidade, é encaminhada para o agendamento de primeira vez na deliberação cirúrgica (no caso de tumores operáveis) ou na oncologia clínica (no caso de tumores inoperáveis).

A partir da matrícula no $\mathrm{HC}$ III, a cliente passa a fazer parte de um fluxo no qual inúmeras vezes terá contato com a enfermeira. Assim, estará com a enfermeira no Grupo de orientação pré-operatória; na consulta de enfermagem na internação; no Grupo de orientação da internação cirúrgica; no Grupo de orientação na alta; no grupo Pro-Mama; na consulta de enfermagem na sala de curativos; na consulta de enfermagem de primeira vez na quimioterapia; na consulta de enfermagem na pesquisa clínica; e na consulta de enfermagem pré-radioterapia (que está em fase de formulação).

Dentre as diversas atribuições da enfermeira no HC III, portanto, destacam-se a atividade de consulta de enfermagem e de orientação à clientela e seus familiares. 
Essas orientações são feitas através da consulta de enfermagem, de palestras com recursos audiovisuais, de folders, algumas vezes, em conjunto com a equipe multiprofissional, e são direcionadas à vivência dos diversos tipos de modalidades terapêuticas para o câncer de mama.

Cabe ressaltar que, apesar do direcionamento técnico e científico da consulta de enfermagem e das orientações, há também a possibilidade de se olhar essa cliente sob o ponto de vista que considera o serdo humano.

Então, ao analisar compreensivamente a prática da enfermagem nos diversos momentos pelos quais a cliente passa durante o seu tratamento, foi possível apreender que os objetivos tanto da consulta de enfermagem como das orientações foram direcionados para uma prática técnica, científica e humana, com o intuito de alcançar o que se mostrou como possibilidade de ser$c^{*}{ }^{*}$ e junto-à cliente durante seu tratamento e seguimento.

Entendo, portanto, que devemos olhar a cliente, a pessoa, não apenas como objeto do assistir, mas como sujeito do nosso cuidado que, sendo, merece um olhar de natureza compreensiva e a oportunidade de recorrer à enfermeira, não para agir ou pensar por ela, mas para auxiliá-la em suas dificuldades, dúvidas, medos e ansiedades, deixando sempre claro que ela tem a responsabilidade final sobre si mesma. Desse modo a enfermeira estará ajudando a cliente a de-cidir-se e assumir seu próprio destino ${ }^{(11)}$.

Nesse processo, a enfermeira, então, está oportunizando a essa cliente a possibilidade de cuidar de si, de reivindicar da Instituição que receba atenção e tratamento que minimize o máximo possível seu sofrimento. E, está, assim, abrindo o caminho para a cliente exercer a sua cidadania, pois, direcionar alguém para o autocuidado, implica em esse alguém conhecer os seus direitos de cidadão e, portanto, de requerer assistência à saúde digna e merecida por qualquer pessoa.

Assim, os objetivos assistenciais visam: informar sobre rotinas hospitalares e procedimentos a serem realizados, diminuindo o stress das clientes gerado pela desinformação; facilitar e/ou possibilitar a recuperação física, emocional e social da cliente, preparando-a para o autocuidado (realização de curativo, cuidados com o dreno, exercícios); dar voz à cliente, permitindo que exponha seus medos, anseios, dúvidas e expectativas; procurar identificar e intervir nos aspectos que poderão prejudicar o tratamento e a recuperação física e moral; auxiliar a cliente e seus familiares a identificar e mobilizar fontes de ajuda para resolução de problemas; facilitar o acesso aos demais profissionais da equipe multidisciplinar; esclarecer sobre a conduta cirúrgica, certificando-se da compreensão e aceitação da mesma pela cliente; permitir que a cliente e seus familiares tomem decisões sobre o tratamento proposto; discutir sobre o retorno às atividades e convívio social, sexualidade, métodos anticoncepcionais e autoexame das mamas e do plastrão; explicar e discutir as alterações decorrentes do ato cirúrgico (mutilação, limitação dos movimentos do membro superior, sintomatologia no pós-operatório, curativos e punção de seroma); orientar e enfatizar os cuidados necessários póslinfadenectomia; identificar e intervir nas possíveis alterações presentes na auto-imagem e na auto-estima (aceitação, negação, rejeição); enfatizar a importância do seguimento; abordar a possibilidade de reconstrução mamária.

\section{O ESPAÇO ASSISTENCIAL - A DIMENSÃO ONTOLÓGICA DO CUIDADO}

O cuidado de enfermagem tem como objetivo, em sua essência, assistir o ser humano em sua totalidade e, portanto, o cliente deve ser visto como um todo, observando-se a relação mente e corpo. Dessa forma, podese perceber que cada indivíduo é singular e tem necessidades e valores próprios.

A atuação da enfermeira no cotidiano do cuidar, portanto, deve se refletir numa assistência de enfermagem de qualidade que direciona para o autocuidado, objetiva a melhoria da qualidade de vida da cliente e, ainda, permite o reconhecimento e a valorização do profissional ao estabelecer relação positiva e empática enfermeiracliente $^{(8)}$.

Ao assistir a mulher que tem um câncer de mama e, em conseqüência, se submete ao tratamento cirúrgico e/ou clínico, pude compreender como no dia a dia criamos laços e compromissos com essa clientela.

A partir do momento em que iniciamos qualquer aproximação com a cliente, mesmo que de caráter puramente informativo, nos tornamos responsáveis por

\footnotetext{
* No pensar de Heidegger, o ser-com é uma característica existencial e deve se exprimir na convivência através de um preocupar-se com o outro e um ocupar-se das coisas
} 
aquele de quem cuidamos. E não apenas responsáveis por conhecer e aplicar, na assistência à pessoa, os princípios técnicos e científicos mais atuais, visando a recuperação da saúde da cliente, mas, também, devemos estar atentos e disponíveis para auxiliar no enfrentamento da doença e suas conseqüências.

Durante quinze anos de convívio com a cliente portadora de câncer de mama e, portanto, com muitas mastectomizadas, pude conhecer e reconhecer vários sinais e sintomas decorrentes do medo nas diversas fases do diagnóstico, tratamento e reabilitação pós-cirúrgica.

O medo é sempre latente nessa clientela, durante toda a história e a trajetória de sua doença e luta pela vida. Medo de ouvir que tem câncer, medo de enfrentar os comentários da família e dos amigos, medo da cirurgia, medo de se ver e se mostrar mutilada, medo das seqüelas da cirurgia, medo de não saber se cuidar e se tornar dependente, medo de fazer o tratamento complementar, medo da doença voltar, medo de não agüentar e morrer.

No entanto, ao longo de sua trajetória, a cliente mostra-se buscando vencer os obstáculos e é nesse momento que a enfermeira tem papel fundamental. Dar voz e possibilitar ao outro que expresse o sentido de enfrentar a mastectomia e o câncer de mama em suas várias etapas e sobre como gostaria de ser ajudado, é imprescindível para o sucesso da reabilitação da cliente.

Esse apoio parece auxiliar na recuperação do equilíbrio necessário ao ser-a $i^{*}$ para continuar a ser o que ele é em sie descobrir suas possibilidades mais próprias. E compreendendo-se como ser possível, o ser-aí pode então de-cidir com liberdade seu próprio modo de ser e ex-sistir frente à doença, ao tratamento, seus efeitos colaterais e sua reablitação(11).

O intuito, portanto, da atuação da enfermeira, é o de poder oferecer assistência que todo ser do humano merece, assistência técnica e científica, humana e singular e que se possa ver o outro como um Ser que possui sentimentos, que pensa, que tem a possibilidade de escolher e de-cidir e não como um organismo doente e dependente.

Portanto, é pensando na reabilitação da mulher que se submeteu à mastectomia ou segmentectomia, que há a realização da orientação individual para o autocuidado, feita pela enfermeira desde que a cliente é internada; do grupo de orientação à mulher e sua família no pré-operatório e quando da alta hospitalar; do grupo Pro-Mama; e das consultas de enfermagem na sala de curativos, quimioterapia, pesquisa clínica e, muito em breve, préradioterapia.

Essas orientações visam esclarecer e apoiar a mulher e sua família para que possam, efetivamente, tomar parte na reabilitação psicossocial e, também, procuram habilitar a cliente ao autocuidado. Pretendem, também, dar estímulo para que readquiram a autoconfiança e mantenham o moral elevado.

As mulheres e seus familiares consideram essas atuações da enfermeira como muito úteis e eficientes, pois, segundo eles, aliviam a tensão, esclarecem as dúvidas e ajudam o fortalecimento psicológico, permitindo que as mulheres enfrentem mais positivamente as adversidades surgidas com o diagnóstico da doença e seu tratamento.

Nesse contexto, a Fenomenologia surge como um caminho que permite a compreensão do outro, no caso a cliente, que teve seu cuidado confiado à enfermeira ${ }^{(12)}$.

O pensar e o agir fundado em base fenomenológica conduz à reflexão sobre o ser-aí, permitido um encontro de natureza existencial com o nosso semelhante, aquele de quem cuidamos e que passa um período difícil de sua vida, quando acometido, por exemplo, pelo câncer de mama. Ao nos conduzir a esse estado reflexivo, abre o caminho para repensar a atividade assistencial ainda hoje sustentada no modelo biologicista/biomédico e que, em conseqüência, se mostra tão afastada da clientela, do ser do humano.

Essa atividade vem sendo centrada nos aspectos biomédicos, na separação do homem em órgãos independentes, nas técnicas, nos custos, na produtividade, nos lucros hospitalares. Assim, parece passar ao largo do doente como pessoa e de suas questões existenciais, levando os profissionais de enfermagem a se afastarem do ser do cliente, privilegiando o quantitativo de atendimentos que elevam os lucros e a produtividade individual. A tais fatos somam-se ainda o cumprimento da carga horária semanal e das escalas de serviço em detrimento da pessoa doente ${ }^{(11)}$.

Então, no meu caminhar, apoiada na Fenomenologia e no pensamento de Martin Heidegger, importou o meu cotidiano assistencial onde apareceram as inquietações iniciais e o olhar atentivo questionador dos fatos técnico-científicos, que não respondiam todas as questões surgidas no meu dia-a-dia profissional. Ao

\footnotetext{
* Heidegger usou essa palavra para designar a existência própria do homem
} 
interrogar o ente mulher com diagnóstico de câncer de mama e submetida à mastectomia emergiu a questão do ser (ser-aí, dasein, presença) e a possibilidade de compreensão e de desvelamento do sentido desse ser do humano, a quem presto assistência de enfermagem.

A partir de minha vivência e experiência profissional, e por entender a enfermagem como uma assistência que se dá ao outro, numa relação entre pessoas, penso a fenomenologia como método adequado para a assistência, a pesquisa e o ensino na enfermagem já que busca a compreensão do humano em seu cotidiano. A investigação fenomenológica possibilita compreender o ser-aí no seu em si mesmo, propiciando, assim, melhor compreensão daquele de quem se cuida $e$, conseqüentemente, a possibilidade de um assistir mais humanizado e holístico porque se realiza a partir do outro, daquela que recebe o cuidado.

Compreendi, em todos esses anos de trabalho na assistência à mulher com câncer de mama, como o apoio, a disponibilidade e a orientação fornecida pela enfermeira à cliente, sobre sua doença, sobre o que esperar após o diagnóstico do câncer, sobre como se dá o tratamento e suas conseqüências e, ainda, a reabilitação são importantes para auxiliar a mulher na superação desses momentos difíceis de sua existência.

Pude ainda perceber como a disponibilidade, as orientações e o apoio às necessidades de cada pessoa na forma de grupo explicativo são, em alguns momentos, ainda não suficientes e não ajustados aos aspectos individuais que envolvem, por exemplo, a observação pela enfermeira dos gestos, das expressões, do olhar, enfim das emoções transmitidas pela cliente já que no coletivo, relevantes são os fatos, aquilo que já sabemos e conhecemos, e que é relativo ao que se observa e se comprova, na maioria das vezes e quase sempre, no acompanhamento terapêutico em oncologia ${ }^{(11-13)}$. Assim, notei como a consulta de enfermagem é importante pois possibilita, em sua relação eu e tu, a singularidade de cada pessoa emergir e, portanto, deve estar permeando a atuação da enfermeira no seu cotidiano assistencial.

Vislumbro a assistência de enfermagem pela ótica da compreensão como possibilidade do agir profissional. Compreensão essa que não tem apenas o significado de entender, notar ou explicar as necessidades fisiológicas emergentes da patologia e do tratamento da cliente, mas que nos permite, enquanto profissionais da saúde que somos, estar com o outro como pessoa em sua totalidade, e não apenas com a sua doença e/ou tratamento. Privilegiando o outro sim, como pessoa, cidadã e objeto de nosso cuidado enquanto enfermeiras, sem nos determos somente no problema físico que, de imediato, o aflige, ou seja, valorizando o exercício da empatia e da cidadania como forma de convivência e assistência diária ao outro ${ }^{(13)}$.

Percebi, então, a possibilidade de olhar o ser humano como ser-aí no meu cotidiano de assistir o outro, ou seja, não olhá-lo apenas a partir da dimensão da experiência, mas, também, da existência. Compreendo que o modelo biomédico, ao qual nossa formação enquanto enfermeiras está amplamente relacionada, entifica o humano pela patologia, tratando mais do que cuidando. Porém, a assistência de enfermagem pode-ser de natureza não apenas científica/explicativa, mas, também, de natureza compreensiva.

Nesse sentido, penso que as enfermeiras devem estar mais atentas à sua atuação no dia-a-dia da labuta, pois parece que muitas vezes não percebem que, ao desenvolver ações educativas em grupo e/ou na consulta de enfermagem, privilegiando a singularidade, estão assumindo o modo de ser sendo-aí-com a cliente e, dessa forma, contribuindo para fortalecer os laços sociais e humanos com a sua clientela no sentido de possibilitá-la a ser o que é em si mesma e tem direito de ser, criando, assim, a possibilidade de uma qualidade de vida melhor.

Cuidar é a essência da enfermagem e implica envolvimento, zelo, amor, compaixão, ética. Cuidar não é tratar apenas de uma doença, mas é também vê-la como possibilidade do ser de quem cuidamos. Portanto, ouvir, tocar, estar disponível é uma forma de humanizar a assistência e resgatar o cuidado que, em nossa cultura científica, foi relegado e colocado em suspeição por ser de natureza subjetiva.

Ainda sobre o cuidado diz-se: "Pelo cuidado não vemos a natureza e tudo que nela existe como objetos. A relação não é sujeito-objeto, mas sujeito-sujeito"(14).

Sob essa ótica, a cliente pode-ser ouvida e assistida mediante um cuidado que não se basta no conhecimento científico e que, portanto, na dimensão ontológica, abre o ser-aípara o seu ser originário que é a cura.

Cura que não significa a resolução de uma patologia, mas, sim, cuidar ou zelar, ou, ainda, relacionarse com algo, e que é considerada por Heidegger, como estrutura fundamental do ser-al ${ }^{(15)}$. 
CONSIDERAÇÕES FINAIS - O CUIDADO DE ENFERMAGEM COMO POSSIBILIDADE

Este artigo buscou refletir sobre a prática assistencial da enfermeira no cotidiano de cuidar de mulheres com câncer de mama, submetidas à mastectomia. Abordou a problemática do câncer de mama a partir de sua incidência e o impacto causado pelo diagnóstico e tratamento cirúrgico dessa patologia, para a mulher. Numa atitude compreensiva, discutiu os aspectos ônticos e ontológicos relativos ao cuidado de enfermagem prestado a essa mulher, num hospital especializado em mastologia oncológica, considerando que esses aspectos permeiam a prática assistencial, e valorizando a dimensão existencial das pessoas envolvidas. Encontrou na Fenomenologia um caminho para o cuidado que é cura, que é singular, que é humanizado e também é direcionado ao resgate do ser-si-mesmo, cuidando de si com responsabilidade.

Finalmente, ancorada na interpretação fenomenológica, é possível tecer considerações sobre as possibilidades do cuidado prestado a essa clientela, propondo que tal cuidado deva ser realizado considerandose a mulher como ser singular, como cidadã, como pessoa responsável por sua saúde e seu autocuidado, como um todo de carne e espírito, mente e corpo, ao qual se deve prestar assistência que congregue técnica, ciência e humanização.

Portanto, a atenção à mulher mastectomizada tem como objetivo final capacitá-la para o autocuidado. Usualmente, em nosso sistema de saúde, que segue o modelo biomédico, envolver a cliente no autocuidado é compreendido apenas como o ato de dar-lhe informações e orientações sobre sua patologia e seu tratamento. Sobre aquilo que a equipe de saúde considera que ela deve

\section{REFERÊNCIAS BIBLIOGRÁFICAS}

1. Ministério da Saúde (BR). Instituto Nacional de Câncer. Estimativa da incidência e mortalidade por câncer no Brasil. Rio de Janeiro (RJ): Instituto Nacional de Câncer; 2001.

2. Carlsson M, Hamrin E. Psychological and psychosocial aspects of breast cancer and breast cancer treatment. Cancer Nurs 1994; 17(5):418-28.

3. Rowland JH, Massie MJ. Psychologic Reactions to Breast Cancer Diagnosis, Treatment and Survival. In: Harris JR, Lippman ME, Morrow M, Hellman S, organizadores. Diseases of the Breast. Philadelphia (PEN): Lippincott-Raven Publishers; 1996. p. 919-38. cumprir para ajudar no tratamento.

No entanto, a cliente deve ser vista como uma pessoa com questões e preocupações singulares. Portanto, a atuação da enfermeira não se reduz a orientações e informações sobre sua doença e seus direitos à saúde, mas envolve também atuação em nível existencial. Dessa forma, a enfermeira sendo-com a equipe de enfermagem, sendo-com a equipe de saúde, sendosujeito de suas ações em ambas e não apenas uma peça da engrenagem assistencial, é possível desenvolver o cuidado holístico e o autocuidado eficiente, já que estará centrado na singularidade de cada um, na sua história de vida e nos seus anseios de futuro.

Esta análise permite apreender que, ao olhar a mulher mastectomizada compreensivamente em sua singularidade, nas consultas de enfermagem, a enfermeira está dando a ela voz para expressar o sentido, ou seja, expressar a compreensão singular que ela tem dessa sua vivência. E é assim que se completa o ciclo que leva ao autocuidado eficiente.

Portanto, a ação assistencial da enfermeira a partir da dimensão existencial da mulher mastectomizada tem a possibilidade de ocorrer num poder-ser, num entrelaçamento de ações e intervenções científicas, sociais e de natureza compreensiva, envolvendo o cliente, a família e as enfermeiras em suas relações de trabalho, despertando, ainda, suas consciências de cidadania e de ser-sujeito de suas ações.

Abre-se, então, a possibilidade da concretude social da enfermagem a partir da enfermeira que se mostra enquanto ser-aíno seu modo próprio de ser, sendo-coma cliente sem dominá-la, preocupando-se com ela e não apenas dela se ocupando. Prestando cuidado denominado por Heidegger de "anteposição liberadora", pois devolve ao Ser a capacidade de cuidar de si.

4. Almeida AM, Mamede MV, Panobianco MS, Prado MAS, Clapis MJ . Construindo o significado da recorrência da doença: a experiência de mulheres com câncer de mama. Rev Latino-am Enfermagem 2001; 9(5):63-9.

5. Suominen T. Breast cancer patients opportunities to participate in their care. Cancer Nurs 1992; 15(1):68-72.

6. Turns DM. Psychosocial Factors In: Donegan WL, Spratt JS, organizadores. Cancer of the Breast. Philadelphia (PEN): W. B. Saunder; 1988. p. 728-38.

7. Silva RM, Mamede MV. Conviver com a Mastectomia. Fortaleza (CE): Universidade Federal do Ceará; 1998.

8. Amorim MH. C. A Enfermagem e a Psiconeuroimunologia no câncer de mama. [tese]. Rio de Janeiro (RJ): Escola de Enfermagem Anna Nery/UFRJ; 1999. 
9. Yabro CH. The Oncology Nurse. In: De Vita VT Jr, Hellman NS, Rosenberg AS, organizadores. Principles and Practice of Oncology. Philadelphia (PEN): J.B. Lippincott; 1997. p. 291723.

10. Ades T, Greene P. Principles of Oncology Nursing. In: Holleb Al, Fink DJ, Murphy GP, organizadores. American Cancer Society Textbook of Clinical Oncology. Atlanta (GEO): American Cancer Society; 1991. p. 587-93.

11. Camargo TC. O ex-sistir feminino enfrentando a quimioterapia para o câncer de mama: um estudo de enfermagem na ótica de Martin Heideqger. [tese]. Rio de Janeiro (RJ): Escola de Enfermagem Anna Nery/UFRJ; 2000. 12. Lopes RLM, Souza IEO. A fenomenologia como abordagem metodológica: compartilhando a experiência de mulheres que buscam a prevenção do câncer cérvico-uterino. Rev Latino-am Enfermagem 1997; 5(3):5-11.

13. Camargo TC, Souza IEO. Enfermagem à mulher em tratamento quimioterápico: uma análise compreensiva do assistir. Rev Bras Enfermagem 1998 julho/setembro; 51(3):357-68.

14. Boff L. Saber Cuidar: ética do humano/compaixão pela terra. 3aㅡ ed. Petrópolis (RJ): Vozes; 1999.

15. Heidegger M. Todos nós...ninguém. São Paulo (SP): Editora Moraes; 1981. 